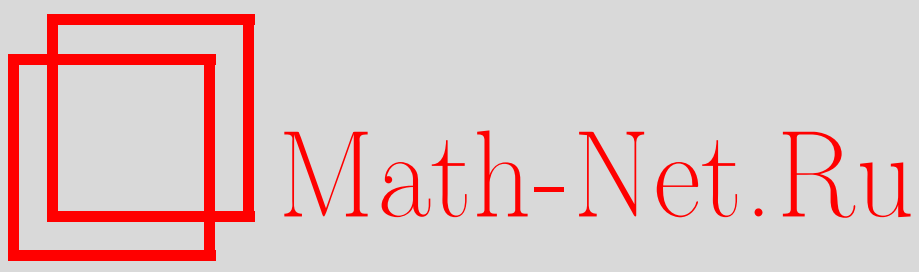

В. И. Скалыга, О гомотопическом методе в многокритериальных бесконечномерных задачах, Изв. РАН. Сер. матем., 1997, том 61, выпуск 4, 137-154

DOI: https://doi.org/10.4213/im139

Использование Общероссийского математического портала Math-Net.Ru подразумевает, что вы прочитали и согласны с пользовательским соглашением http: //www. mathnet.ru/rus/agreement

Параметры загрузки:

IP: 54.209 .52 .79

26 апреля 2023 г., 15:49:28 
УДК 517.977 .58

\title{
В.И. Скалыга
}

\section{О гомотопическом методе в многокритериальных бесконечномерных задачах}

\author{
Устанавливается гомотопическая инвариантность оптимума в многокритери- \\ альных задачах. \\ Библиограффия: 14 наименований.
}

\section{§1. Введение}

Гомотопический метод исследования оптимизационных задач был предложен в работах [1]-[5]. В них исследовались безусловная минимизация гладких функционалов в гильбертовом пространстве и гладкие и негладкие конечномерные задачи на безусловный и условный минимум.

В этих работах выделены классы оптимизационных задач, зависящих от параметра и обладающих следуюшим свойством: если в процессе деформации оптимизационной задачи ее экстремаль изолирована и при каком-либо значении параметра является точкой минимума, то она является точкой минимума соответствующих оптимизационных задач при всех значениях параметра.

Этот общий принцип, названный деформационным принципом минимума, нашел многочисленные применения в анализе, вариационном исчислении, теории устойчивости, математической физике и т.д.

В дальнейшем деформационный принцип минимума был обобщен на бесконечномерные задачи математического программирования и бесконечномерные негладкие оптимизационные задачи (см. [6]-[11]).

В настоящей работе этот деформационный принцип обобщается для многокритериальных оптимизационных задач в банаховом пространстве. При этом число критериев может быть бесконечно.

\section{§2. Деформационная теорема}

Пусть $X$ и $Y$-вешественные банаховы пространства с соответственно заданными нормами $|\cdot|$ и $|\cdot|_{Y}$. Пусть задано отображение $F(x): X \rightarrow Y$, имеюшее строгую производную $D_{S} F(x)$ в каждой точке $x \in X$. Пусть на $Y$ задан конус $K$, определяюший частичную упорядоченность на $Y$ : для $u, v \in Y \quad u \leqslant v$, если $v-u \in K$, и $u<v$, если $u \leqslant v$ и $u \neq v$.

Работа выполнена при финансовой поддержке РФФИ (грант № 96-01-00767).

(C) В.И. СКАЛЫГА 1997 
Будем называть точку $x_{*} \in X$ локально $K$-оптимальной точкой отображения $F(x)$, если сушествует окрестность $V$ точки $x_{*}$, все точки которой не удовлетворяют неравенству

$$
F(x)<F\left(x_{*}\right) .
$$

(Если $Y$ конечномерно и $K$ - конус векторов с неотрицательными координатами, то $K$-оптимальность называют оптимальностью по Парето [12].)

Точку $x_{*} \in X$ будем называть локально оптимальной точкой отображения $F(x)$, если сушествует окрестность $V$ точки $x_{*}$, для всех точек которой вьполнено неравенство

$$
F\left(x_{*}\right) \leqslant F(x) .
$$

Если в этих определениях положить $V=X$, то соответственно получим определения $K$-оптимальной точки и оптимальной точки отображения $F(x)$.

Задачу нахождения локального $K$-оптимума отображения $F(x)$ будем обозначать

$$
F(x) \rightarrow K-\text { opt. }
$$

В настоящей работе изучается гомотопическая инвариантность решений задач (1). Для формулировки основного результата нам потребуется понятие обобщенного градиента Кларка.

Пусть $f(x)$ - локально липшицев функционал, определенньй на $X$. Обобщенной производной функционала $f$ по направлению $v$ называется [12] число

$$
f^{0}(x ; v)=\lim _{y \rightarrow x} \sup _{t \rightarrow+0} t^{-1}(f(y+t v)-f(y)) .
$$

Обобщенным градиентом $\partial f(x)$ функционала $f$ в точке $x$ называется [12] такое множество в $X^{*}$, что при $\xi \in \partial f(x)$ и всех $v \in X$ выполнено неравенство

$$
f^{0}(x ; v) \geqslant\langle\xi, v\rangle \text {. }
$$

Обобщенный градиент является непустым выпуклым слабо* компактным множеством в $X^{*}$, а обобщенная производная $f^{0}(x ; v)$ полунепрерывна сверху как функция $(x, v)$ и как функция $v$ является опорной функцией обобщенного градиента $\partial f(x)$ [12]. Точка $x_{*}$ называется критической точкой функционала $f$, если $0 \in \partial f\left(x_{*}\right)$.

Липшицев функционал $f(x)$ называется [12] регулярным в точке $x$, если для каждого $v \in X$ обобщенная производная $f^{0}(x ; v)$ совпадает с классической производной $f^{\prime}(x ; v)$ по направлению $v$.

Пусть $\stackrel{\circ}{B}(R)=\{x \in X:|x|<R\}-$ шар в $X$. Будем говорить, что функционал $f$ удовлетворяет условию $($ ПС) Пале и Смейла $[13]$ на $\stackrel{\circ}{B}(R)$, если для каждого замкнутого множества $S \subset \stackrel{\circ}{B}(R)$ справедливо следующее свойство: если $0 \notin \partial f(x)$ для каждого $x \in S$, то

$$
\inf _{y \in \partial f(x), x \in S}|y|_{X^{*}}>0
$$


Класс функционалов, удовлетворяюших условию (ПС), достаточно широк. Например, ему принадлежат функционалы, определенные на рефлексивном банаховом пространстве и удовлетворяюшие условию $(S)$ : если $x_{n}$ слабо сходится к $x_{0}$ и

$$
\varlimsup_{n \rightarrow \infty} \inf _{y \in \partial f\left(x_{n}\right)}\left\langle y, x_{n}-x_{0}\right\rangle \leqslant 0,
$$

то $x_{n}$ сильно сходится к $x_{0}$. Такие функционалы называются $X$-правильнылми; для гладких задач они исследовались в [1], [2], [6], [7]. Суммы $X$-правильного и $X$-правильного или выпуклого функционалов являются $X$-правильными функционалами.

Рассмотрим множества

$$
K^{*}=\left\{p \in Y^{*}: p(u) \geqslant 0 \quad \forall u \in K\right\}, \quad \mathfrak{P}=\left\{p \in K^{*}:|p|_{Y^{*}}=1\right\}
$$

и функционал

$$
f(x)=\sup _{p \in \mathfrak{P}} p\left(F(x)-F\left(x_{*}\right)\right),
$$

где $x_{*}$ - фиксированный элемент из $X$. Функционал $f(x)$ является локально липшицевым. Ниже будет доказана формула для обобшенного градиента Кларка

$$
\partial f(x)=\overline{\mathrm{co}}\left\{\xi=\operatorname{li}_{i \rightarrow \infty} \operatorname{i.m}_{\infty}\left(D_{S} F(x)\right)^{*} p_{i}: p_{i} \in \mathfrak{P}, \quad \lim _{i \rightarrow \infty} p_{i}\left(F(x)-F\left(x_{*}\right)\right)=f(x)\right\},
$$

где l.i.m - обозначение слабого* предела последовательности $\xi_{i} \in X^{*}$; $\left(D_{S} F(x)\right)^{*}$ - оператор, сопряженный к $D_{S} F(x)$; $\overline{\text { со }}$ - слабо* замкнутая выпуклая оболочка.

Если точка $x_{*}$ является локально $K$-оптимальной точкой задачи $(1)$, то в силу теоремы отделимости она будет точкой локального минимума $f(x)$. Тогда в силу (2) и (3) будет выполнено включение

$$
0 \in \overline{\mathrm{co}}\left(\left(D_{S} F\left(x_{*}\right)\right)^{*} p: p \in \mathfrak{P}\right)
$$

Точку $x_{*}$, для которой удовлетворяется включение (4), будем называть критической точкой отображения $F(x)$.

В дальнейшем шар $B(1)=\bar{B}(1)$ будем обозначать просто через $B$.

Однопараметрическое семейство отображений $F(x ; \lambda): X \rightarrow Y$ и семейство конусов $K(\lambda) \subset Y(0 \leqslant \lambda \leqslant 1)$ назовем $\stackrel{\circ}{B}(R)$-невырожденной деформачией отображения $F(x ; 0)$ и конуса $K(0)$ в отображение $F(x ; 1)$ и конус $K(1)$, если:

1) отображение $F(x ; \lambda)$ строго дифференцируемо по $x$ на $B(R)=\bar{B}(R)$ при любом $\lambda \in[0,1]$ и по $\lambda$ равномерно непрерывно относительно $x \in B\left(R_{1}\right) \subset \stackrel{\circ}{B}(R)$;

2 ) отображение $D_{S} F(x ; \lambda): B(R) \times[0,1] \rightarrow \mathscr{L}(X, Y)$ равномерно ограничено по норме при каждом $\lambda \in[0,1]$ относительно $x \in B\left(R_{1}\right) \subset \stackrel{\circ}{B}(R)$ и по $\lambda$ равномерно непрерывно относительно $x \in B\left(R_{1}\right) \subset \stackrel{\circ}{B}(R)$; 
3) многозначное отображение $\lambda \rightarrow K(\lambda) \cap B_{Y}:[0,1] \rightarrow Y$ полунепрерьвно сверху, т.е. для каждого $\varepsilon>0$ и $\lambda_{0} \in[0,1]$ найдется окрестность $N\left(\lambda_{0}\right) \subset[0,1]$ точки $\lambda_{0}$ такая, что для точек $\lambda \in N\left(\lambda_{0}\right)$ выполнено включение

$$
K(\lambda) \cap B_{Y} \subset K\left(\lambda_{0}\right) \cap B_{Y}+\varepsilon B_{Y}
$$

4) многозначное отображение $\lambda \rightarrow K(\lambda) \cap B_{Y}:[0,1] \rightarrow Y$ усиленно полунепрерывно снизу, а именно, для каждого $\lambda_{0} \in[0,1]$ и $\varepsilon>0$ найдется окрестность $N\left(\lambda_{0}\right) \subset[0,1]$ точки $\lambda_{0}$ такая, что для точек $\lambda \in N\left(\lambda_{0}\right)$ выполняется соотношение

$$
\left(u+\varepsilon B_{Y}\right) \cap K(\lambda) \cap B_{Y} \neq \varnothing \quad \forall u \in K\left(\lambda_{0}\right) \cap B_{Y}
$$

$5)$ при каждом $\lambda \in[0,1]$ у отображения $F(x ; \lambda)$ при конусе $K(\lambda)$ существует критическая точка $x_{*}(\lambda) \in \stackrel{\circ}{B}\left(R_{1}\right)$, непрерывно зависящая от $\lambda$;

6) при каждом $\lambda \in[0,1]$ функционал

$$
f(x ; \lambda)=\sup \left\{p\left(F(x ; \lambda)-F\left(x_{*}(\lambda), \lambda\right)\right): p \in K^{*}(\lambda), \quad|p|_{Y^{*}}=1\right\}
$$

удовлетворяет условию $\left(\right.$ ПС) на $\stackrel{\circ}{B}(R)$ и на множестве $\stackrel{\circ}{B}(R) \backslash\left\{x_{*}(\lambda)\right\}$ не имеет критических точек.

ТЕОРема 1. Пусть существует $\stackrel{\circ}{B}(R)$-невырожденная деформация отображения $F(x ; 0)$ и конуса $K(0)$ в отображение $F(x ; 1)$ и конус $K(1)$. Пусть $x_{*}(0)$ является точкой локального $K$-оптимума отображсения $F(x ; 0)$. Тогда $x_{*}(1)$ является точкой локального $K$-оптимума отображения $F(x ; 1)$. Если на $B(R) \backslash\left\{x_{*}(1)\right\}$ функциональ $p(F(x ; 1)), \quad p \in \mathfrak{P}(1)$, не имеют критических точек и удовлетворяют условию $\left(\right.$ ПС), то $x_{*}(1)$ является локально оптимальной точкой отображения $F(x ; 1)$.

Доказательству теоремы 1 предпошлем несколько лемм. При доказательстве лемм и теоремы, не уменьшая обшности, будем полагать, что $x_{*}(\lambda) \equiv 0$ и $F(0, \lambda) \equiv 0$ при $\lambda \in[0,1]$.

Лемма 1. Пусть $\lambda_{0} \in[0,1]$ u $f(x)=f\left(x, \lambda_{0}\right)$. Тогда $f(x)$ - регулярный функционал и для градиента Кларка $f(x)$ имеет место формула (3).

ДокАЗАТЕЛЬСТво. Для доказательства совпадения двух слабо* замкнутых выпуклых множеств достаточно доказать равенство их опорных функций

$$
f^{0}(x ; v)=\sup _{\xi \in A}\langle\xi, v\rangle \quad \forall v \in X
$$

где $A$ - множество в правой части равенства (3).

Пусть $v$ фиксировано и $y_{i} \rightarrow x, t_{i} \downarrow 0$ - такие последовательности, что последовательность

$$
\Delta_{i}=\left(f\left(y_{i}+t_{i} v\right)-f\left(y_{i}\right)\right) t_{i}^{-1}
$$


сходится к $f^{0}(x ; v)$. Пусть $\mathfrak{P}=\left\{p \in K^{*}\left(\lambda_{0}\right):|p|_{Y^{*}}=1\right\}, p_{i} \in \mathfrak{P}$ и

$$
\varepsilon_{i} t_{i}+p_{i}\left(F\left(y_{i}+t_{i} v\right)\right) \geqslant f\left(y_{i}+t_{i} v\right) \quad \forall i>0,
$$

где $\varepsilon_{i} \downarrow 0$. Тогда

$$
\Delta_{i} \leqslant\left(p_{i}\left(F\left(y_{i}+t_{i} v\right)-p_{i}\left(F\left(y_{i}\right)\right)\right) t_{i}^{-1}+\varepsilon_{i} .\right.
$$

Пусть последовательность $p_{i}$ слабо* сходится к $p$. Так как $\left(F\left(y_{i}+t_{i} v\right)-F\left(y_{i}\right)\right) t_{i}^{-1}$ сходится к $D_{S} F(x) v$, а $p_{i}(F(x))$ сходится к $f(x)$, то из (6) следует

$$
f^{0}(x ; v) \leqslant \sup _{\xi \in A}\langle\xi, v\rangle \quad \forall v \in X .
$$

Докажем теперь регулярность $f(x)$ и неравенство, противоположное неравенству (7). Пусть последовательность $t_{i} \downarrow 0$ такая, что

$$
\alpha=\lim _{i \rightarrow \infty}\left(f\left(x+t_{i} v\right)-f(x)\right) t_{i}^{-1}=\lim _{t \downarrow 0} \inf (f(x+t v)-f(x)) t^{-1} .
$$

Чтобы доказать регулярность $f$ в $x$, достаточно показать, что $f^{0}(x ; v) \leqslant \alpha$. Пусть $p_{j}$-произвольная последовательность такая, что $p_{j} \in \mathfrak{P}$ при всех $j>0, p_{j}$ слабо* сходится к $p$ и $\lim _{j \rightarrow \infty} p_{j}(F(x))=f(x)$. Тогда

$$
\begin{gathered}
\alpha_{i}=\left(f\left(x+t_{i} v\right)-f(x)\right) t_{i}^{-1} \geqslant\left(p_{j}\left(F\left(x+t_{i} v\right)\right)-\lim _{j \rightarrow \infty} p_{j}(F(x))\right) t_{i}^{-1}, \\
\alpha_{i} \geqslant \lim _{j \rightarrow \infty} p_{j}\left(F\left(x+t_{i} v\right)-F(x)\right) t_{i}^{-1} .
\end{gathered}
$$

В силу известной теоремы анализа имеют место равенства

$$
\begin{gathered}
\lim _{\substack{j \rightarrow \infty \\
i \rightarrow \infty}} p_{j}\left(F\left(x+t_{i} v\right)-F(x)\right) t_{i}^{-1}=\left\langle p, D_{S} F(x) v\right\rangle \\
=\lim _{i \rightarrow \infty} \lim _{j \rightarrow \infty} p_{j}\left(F\left(x+t_{i} v\right)-F(x)\right) t_{i}^{-1} .
\end{gathered}
$$

Поэтому из (7)-(9) следуют неравенства

$$
\alpha \geqslant \sup _{\xi \in A}\langle\xi, v\rangle \geqslant f^{0}(x ; v),
$$

что и требовалось доказать.

Из условий 3) и 4) вытекает, что многозначное отображение $\lambda \rightarrow K^{*}(\lambda) \cap$ $B_{Y}:[0,1] \rightarrow Y^{*}$ полунепрерывно сверху и усиленно полунепрерывно снизу. Отсюда и из условий 1$), 2)$ и леммы 1 вытекают следующие свойства однопараметрического семейства функционалов $f(x, \lambda)$ :

$\left.1^{\circ}\right)$ функционал $f(x ; \lambda)$ липшицев по $x$ на $B(R)$ при любом $\lambda \in[0,1]$ и по $\lambda$ равномерно непрерывен относительно $x \in B\left(R_{1}\right) \subset \stackrel{\circ}{B}(R)$;

$2^{\circ}$ ) многозначное отображение $\partial_{x} f(x ; \lambda): B(R) \times[0,1] \rightarrow X^{*}$ полунепрерывно сверху по $\lambda$ равномерно относительно $x \in B\left(R_{1}\right) \subset \stackrel{\circ}{B}(R)$.

Однопараметрическое семейство функционалов $f(x, \lambda)$, удовлетворяющее условиям $\left.\left.1^{\circ}\right), 2^{\circ}\right)$ и 6$)$, называется $\stackrel{\circ}{B}(R)$-невырожденной деформачией функционала $f(x ; 0)$ в функционал $f(x ; 1)$. 
Лемма 2. Пусть существует $\stackrel{\circ}{B}(R)$-невырожсденная деформация функиионала $f(x ; 0)$ в функиионал $f(x ; 1)$. Пусть точка $x=0$ реализует локальньй минимум функиионала $f(x ; 0)$. Тогда точка $x=0$ реализует локальньй минимум функиионала $f(x ; 1)$.

ДокАЗАТЕЛЬСтво. Не ограничивая обшности, можно считать, что $f(0 ; \lambda) \equiv 0$. Зафиксируем $\lambda_{0} \in[0,1]$. Пусть $f(x)=f\left(x ; \lambda_{0}\right)$. Построим множество $\mathfrak{M}$ непрерывных сплайнов $x(t)$ таких, что $x(t) \in B\left(R_{1}\right)$ при $t \geqslant 0$, функция $f\left(x(t) ; \lambda_{0}\right)$ убывает и каждое звено сплайна является прямолинейным отрезком

$$
x(t)=x\left(t_{n-1}\right)+\left(t-t_{n-1}\right) v_{n-1}, \quad t_{n-1} \leqslant t \leqslant t_{n}, \quad n=1,2, \ldots
$$

Из условий $2^{\circ}$ ) и 6$) \stackrel{\circ}{B}(R)$-невырожденной деформации вытекает неравенство

$$
m(s)=\inf _{y \in \partial f(x ; \lambda), R_{1} \geqslant|x| \geqslant s>0, \lambda \in[0,1]}|y|_{X^{*}}>0 .
$$

В силу теоремы отделимости [14] для каждого $x \in B\left(R_{1}\right) \backslash\{0\}$ найдется $v(x) \in X$ такой, что $|v(x)|=1$ и

$$
f^{0}(x ; v(x)) \leqslant-m(|x|) c, \quad 1 / 2<c<1 .
$$

В силу полунепрерьвности сверху $f^{0}(x ; v)$ по $x$ найдется число $r(x, v(x))$ такое, что для $u \in B(x, r(x, v(x)))$

$$
f^{0}(u ; v(x)) \leqslant-m(|x|) / 2 .
$$

Обозначим через $S(x)$ множество таких $v$, для которых $|v|=1$ ипри $t \in[0, t(v))$, $x+t v \in B\left(R_{1}\right)$ выполняется неравенство

$$
f^{0}(x+t v ; v) \leqslant-m(k|x+t v|) / 2, \quad k=3 / 4 .
$$

(Через $t(v)$ обозначен максимальный правый конец таких отрезков.) Сплайн $x(t) \in \mathfrak{M}$ с началом в точке $x_{0} \in B\left(R_{1}\right) \backslash\{0\}$ построим следуюшим образом. В качестве направления $v_{0}$ выберем вектор $v_{0} \in S\left(x_{0}\right)$, для которого

$$
t\left(v_{0}\right) \geqslant \sup _{v \in S\left(x_{0}\right)} t(v) / 2
$$

и длина первого отрезка сплайна будет равняться $t\left(v_{0}\right)$. Для следуюших звеньев сплайна поступаем аналогично. Если сплайн $x(t)$ пересекает в момент времени $T$ границу шара $B\left(R_{1}\right)$, то сплайн в этой точке заканчивается.

Докажем следующее 
УТВЕРЖДЕНИЕ 1. Если значения сплайна $x(t) \in \mathfrak{M}$ на конечном отрезке $[0, T]$ принадлежсат $\stackrel{\circ}{B}\left(R_{1}\right)$ u $x(T) \neq 0$, то на отрезке $[0, T]$ нет предельной точки узлов сплайна.

В предположении противного найдется последовательность узлов $t_{n} \rightarrow t_{*}$ при $n \rightarrow \infty$ и для точки $x\left(t_{*}\right)$ сушествуют радиус $r\left(x\left(t_{*}\right)\right)<\left|x\left(t_{*}\right)\right| / 3$ и направление $v\left(x\left(t_{*}\right)\right)$ такие, что для точек шара $B\left(x\left(t_{*}\right), r\left(x\left(t_{*}\right)\right)\right)$ выполнено неравенство (10). Но тогда при достаточно больших $n$ точки $x\left(t_{n}\right)$ принадлежат шару $B\left(x\left(t_{*}\right), r\left(x\left(t_{*}\right)\right) / 8\right)$, что противоречит конструкции сплайна, так как при этих $n$ не вьполняется неравенство (12).

Докажем еще одно вспомогательное

УТВЕРЖДЕНИЕ 2. Пусть при $\lambda_{0} \in[0,1]$ в точке $x=0$ функционал $f(x)=$ $f\left(x ; \lambda_{0}\right)$ имеет строгий минимум на шаре $B(\rho)$. Тогда для числа $\rho_{0}$, где $0<\rho_{0}<\rho$, справедливо неравенство

$$
\inf _{|x|=\rho_{0}} f(x)>0
$$

В предположении противного на сфере $|x|=\rho_{0}$ найдется точка $x_{0}$, для которой вьполнено неравенство

$$
f\left(x_{0}\right)<\delta m\left(k\left(\rho_{0}-\delta\right)\right) / 4
$$

где $0<\delta<\rho_{0}<\rho_{0}+\delta<\rho$. Пусть сплайн $x(t) \in \mathfrak{M}_{\text {и }} x(0)=x_{0}$. Рассмотрим функцию $\varphi(t)=f(x(t))$. Она почти всюду дифференцируема в классическом смысле и на отрезке сплайна $\left(t_{n-1}, t_{n}\right)[12]$

$$
\dot{\varphi}(t) \in\left\langle\partial f\left(x_{n-1}+t v_{n-1}\right), v_{n-1}\right\rangle .
$$

По построению сплайна $x(t)$ множество чисел в правой части включения (14) ограничено сверху числом $-m\left(k\left|x_{n-1}+t v_{n-1}\right|\right) / 2$. Следовательно, сплайн $x(t)$ достигает в некоторый момент времени $t_{*}$ границы шарового слоя $\rho_{0}-\delta \leqslant|x| \leqslant \rho_{0}+\delta$. Но тогда из (13) и (14) вытекает

$$
f\left(x\left(t_{*}\right)\right)=\varphi\left(t_{*}\right)=f\left(x_{0}\right)+\int_{0}^{t_{*}} \dot{\varphi}(\tau) d \tau<-\delta m\left(k\left(\rho_{0}-\delta\right)\right) / 4
$$

что противоречит условию минимума в точке $x=0$ функционала $f(x)$ на $B(\rho)$.

Пусть $\Lambda$ - множество тех $\lambda \in[0,1]$, для которых $x=0$ является точкой локального минимума функционала $f(x ; \lambda)$. Множество $\Lambda$ непусто. Покажем, что оно открыто. Пусть $\lambda_{0} \in \Lambda$. В силу того, что на множестве $\stackrel{\circ}{B}(R) \backslash\{0\}$ нет критических точек функционала $f(x ; \lambda)$, точка $x=0$ является точкой его строгого минимума на некотором шаре $B(\rho)$. Следовательно,

$$
\inf _{|x|=\rho_{0}} f\left(x ; \lambda_{0}\right)=a>0, \quad 0<\rho_{0}<\rho .
$$


Тогда для некоторого $\varepsilon>0$ при $\lambda \in\left[\lambda_{0}-\varepsilon, \lambda_{0}+\varepsilon\right] \cap[0,1]$

$$
\inf _{|x|=\rho_{0}} f(x ; \lambda)>a / 2 .
$$

Зафиксируем $\lambda_{1} \in\left[\lambda_{0}-\varepsilon, \lambda_{0}+\varepsilon\right] \cap[0,1]$. Пусть $f(x)=f\left(x ; \lambda_{1}\right)$. Рассмотрим шар $B\left(\rho_{1}\right)$, для которого

$$
\sup _{|x| \leqslant \rho_{1}} f(x)<a / 2
$$

Тогда сплайн $x(t) \in \mathfrak{M}$ с началом в точке $x_{0} \in B\left(\rho_{1}\right)$ в силу $(15),(16)$ не выходит из шара $B\left(\rho_{0}\right)$ и для функции $\varphi(t)=f(x(t))$ в силу $(11)$ найдется последовательность $t_{k}^{\prime} \rightarrow \infty$, для которой $\dot{\varphi}\left(t_{k}^{\prime}\right) \rightarrow 0$ и $x\left(t_{k}^{\prime}\right) \rightarrow 0$. Следовательно, функционал $f\left(x ; \lambda_{1}\right)$ имеет в точке $x=0$ строгий минимум на шаре $B\left(\rho_{1}\right)$, т.е. множество $\Lambda$ открыто. Установим теперь замкнутость $\Lambda$. Каждому сплайну $x(t) \in \mathfrak{M}$, числу $\varepsilon>0$ и отрезку $[0, T]$ поставим в соответствие множество сплайнов $y(t)$ таких, что при $t \in[0, T]|y(t)-x(t)|<\varepsilon$, узлы сплайнов $x(t)$ и $y(t) \quad\left(t_{n} \in[0, T]\right)$ и направления звеньев совпадают. При $t>T$ сплайны $y(t)$ продолжаются по той же конструкции, что и сплайны $x(t) \in \mathfrak{M}$. При малых $\varepsilon$ для $y(t)$ на отрезке $\left(t_{n-1}, t_{n}\right) \in[0, T]$ выполнено неравенство

$$
f^{0}\left(y(t) ; v_{n-1}\right) \leqslant-m(|y(t)| / 2) / 4
$$

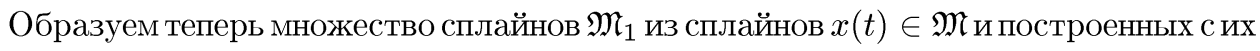
помошью для каждого $x(t)$ отрезка $[0, T]$ и $\varepsilon>0$ сплайнов $y(t)$, удовлетворяющих неравенству (17). По построению сплайны из $\mathfrak{M}_{1}$ обладают аналогом непрерывной зависимости от начальных значений. В силу свойства $\left.2^{\circ}\right) \stackrel{\circ}{B}(R)$-невырожденной деформации справедливо неравенство

$$
\sup _{y \in \partial f(x ; \lambda), x \in B\left(R_{1}\right), \lambda \in[0,1]}|y|_{X^{*}}=M<\infty .
$$

Покажем, что при любом $\lambda \in \Lambda$ для точек шара $B\left(\rho_{1}\right), \rho_{1}=R_{1} m\left(R_{1} / 4\right) /(16 M)$, выполняется неравенство $f(x ; \lambda) \geqslant f(0 ; \lambda)$. Этим замкнутость $\Lambda$ будет доказана. Зафиксируем $\lambda \in \Lambda$. Тогда точка $x=0$ реализует строгий минимум функционала $f(x)=f(x ; \lambda)$ на некотором шаре $B(\rho)$ и, как было показано выше, сушествует непустое максимальное множество $\Omega$, из точек которого сплайны $x(t) \in \mathfrak{M}_{1}$ сходятся к нулю на бесконечности. Пусть $\stackrel{\circ}{B}(r)$ - максимальный открытый шар такой, что $\stackrel{\circ}{B}(r) \subset \Omega$. Для доказательства замкнутости $\Lambda$ достаточно установить оценку $r \geqslant \rho_{1}$. Эта оценка очевидна, если $r \geqslant R_{1} / 2$. Пусть $r<R_{1} / 2$. Рассмотрим на сфеpe $|x|=r$ точку $y_{0} \notin \Omega$ и сплайн $y(t) \in \mathfrak{M}_{1}, y(0)=y_{0}$, не сходяшийся на бесконечности к нулю. Так как функция $f(y(t))$ квалифицированно убывает, то найдется $t_{0}^{\prime}>0$, при котором $3 R_{1} / 4<\left|y\left(t_{0}^{\prime}\right)\right|<R_{1}$. В силу конструкции множества сплайнов $\mathfrak{M}_{1}$ найдется сплайн $x(t) \in \mathfrak{M}_{1}$ с начальным значением $x(0) \in \stackrel{\circ}{B}(r)$, для которого $3 R_{1} / 4<\left|x\left(t_{0}^{\prime}\right)\right|<R_{1}$. Так как $x(0) \in \Omega$, то найдутся такие $t_{1}^{\prime}$ и $t_{2}^{\prime}, t_{1}^{\prime}<t_{2}^{\prime}$, 
что $\left|x\left(t_{1}^{\prime}\right)\right|=3 R_{1} / 4,\left|x\left(t_{2}^{\prime}\right)\right|=R_{1} / 2$ и $R_{1} / 2<|x(t)|<3 R_{1} / 4$ при $t_{1}^{\prime}<t<t_{2}^{\prime}$. Очевидно,

$$
f(x(0))-f(0) \leqslant M|x(0)|<M r .
$$

С другой стороны,

$$
f(x(0))-f(0)>f\left(x\left(t_{1}^{\prime}\right)\right)-f\left(x\left(t_{2}^{\prime}\right)\right)=-\int_{t_{1}^{\prime}}^{t_{2}^{\prime}} \dot{\varphi}(\tau) d \tau
$$

где $\varphi(t)=f(x(t))$. Из неравенств (17) и (19) следует

$$
f\left(x\left(t_{1}^{\prime}\right)\right)-f\left(x\left(t_{2}^{\prime}\right)\right) \geqslant\left(t_{2}^{\prime}-t_{1}^{\prime}\right) m\left(R_{1} / 4\right) / 4 \geqslant R_{1} m\left(R_{1} / 4\right) / 16 .
$$

Из неравенств (18) и (20) вытекает оценка $r>\rho_{1}$. Замкнутость $\Lambda$ доказана. Но тогда $\Lambda=[0,1]$. Лемма доказана.

ДоКАЗАТЕЛЬСТВо ТЕОРЕмЫ 1. Для доказательства первого утверждения теоремы достаточно показать, что точка 0 является точкой строгого локального минимума функционала $f(x ; 1)$. Но точка 0 является точкой локального минимума функционала $f(x ; 0)$. Поэтому первое утверждение теоремы 1 следует из леммы 2.

Докажем второе утверждение теоремы. В силу отсутствия критических точек у функционалов $p(F(x ; 1)), p \in \mathfrak{P}(1)=\left\{\xi \in K^{*}(1),|\xi|_{Y^{*}}=1\right\}$, на множестве $B\left(R_{1}\right) \backslash\{0\}$ и условия (ПС) можно построить для каждого $p(F(x ; 1)), p \in \mathfrak{P}(1)$, на $B\left(R_{1}\right) \backslash\{0\}$ множество сплайнов $\mathfrak{M}_{p}$, аналогичное множеству сплайнов $\mathfrak{M}$, построенного для функционала $f(x)$ при доказательстве леммы 2. Функционал $f(x)=$ $\sup p(F(x ; 1)), p \in \mathfrak{P}(1)$, имеет локальный минимум в нуле. Поэтому найдутся два шара $B\left(\rho_{1}\right) \subset B\left(\rho_{0}\right)$ такие, что для любого $p \in \mathfrak{P}(1)$ и $x_{0} \in B\left(\rho_{1}\right) \backslash\{0\}$ сплайн $x(t) \in \mathfrak{M}_{p}$ с началом в точке $x_{0}$ не выходит за пределы шара $B\left(\rho_{0}\right)$. В силу условия (11) отсюда следует, что сплайн $x(t)$ сходится на бесконечности к нулю. Поэтому для любого $p \in \mathfrak{P}(1)$ и $x_{0} \in B\left(\rho_{1}\right) \backslash\{0\} p\left(F\left(x_{0} ; 1\right)\right)>0$, а это означает, что $F\left(x_{0} ; 1\right)>0$. Теорема доказана.

\section{§3. Многокритериальные задачи с ограничениями}

Рассмотрим задачу $K$-оптимизации с ограничениями

$$
\begin{gathered}
F(x) \rightarrow K-\text { opt } \\
\psi(x) \leqslant 0, \\
x \in C,
\end{gathered}
$$

где отображение $F(x)$ описано в $\S 2, \psi(x)$ - липшицев функционал на каждом шаре в $X, C$ - замкнутое выпуклое множество.

Заметим, что к задаче (21)-(23) сводится формально более общая задача $K$-оптимизации с $n$ ограничениями типа неравенств $\psi_{i}(x) \leqslant 0, i=1, \ldots, n$, следуюшей заменой: $\psi(x)=\max \psi_{i}(x), 1 \leqslant i \leqslant n$. 
Пусть $Q=\{x: \psi(x) \leqslant 0\}, D=Q \cap C$. Для задачи (21)-(23) естественным образом вводятся понятия локально $K$-оптимальной и локально оптимальной точек, а также $K$-оптимальной и оптимальной точек. Точку $x_{*} \in D$ назовем әкстремалью задачи (21)-(23), если

$$
0 \in \overline{\mathrm{CO}}\left(\left(D_{S} F\left(x_{*}\right)\right)^{*} p: p \in \mathfrak{P}\right)+\mu \partial \psi\left(x_{*}\right)+\alpha \partial d_{C}\left(x_{*}\right), \quad \mu \psi\left(x_{*}\right)=0,
$$

где $\mu, \alpha \geqslant 0, d_{C}(x)=\inf _{c \in C}|x-c|$.

Однопараметрическое семейство задач

$$
\begin{aligned}
& F(x ; \lambda) \rightarrow K-\text { opt }, \quad K(\lambda) \subset Y, \\
& \psi(x ; \lambda) \leqslant 0, \\
& x \in C(\lambda), \quad x \in X, \quad \lambda \in[0,1],
\end{aligned}
$$

назовем невырожденной деформачией задачи

$$
\begin{gathered}
F(x ; 0) \rightarrow K-\text { opt }, \quad K(0) \subset Y, \\
\psi(x ; 0) \leqslant 0, \\
x \in C(0)
\end{gathered}
$$

в задачу

$$
\begin{gathered}
F(x ; 1) \rightarrow K-\text { opt }, \quad K(1) \subset Y, \\
\psi(x ; 1) \leqslant 0, \\
x \in C(1),
\end{gathered}
$$

если:

А) отображения $F(x ; \lambda)$ и конусы $K(\lambda)$ удовлетворяют условиям 1$)-4) \S 2$ (при этом $B(R)=X)$;

В) при каждом $\lambda \in[0,1] C(\lambda)$ - замкнутое выпуклое множество;

C) функционалы $\psi(x ; \lambda)$ и $d_{C(\lambda)}(x)$ по $\lambda$ равномерно непрерывны относительно $x$ из каждого шара $B(r) \subset X$, а функционал $\psi(x ; \lambda)$ липшицев по $x$ на каждом шаре $B(r) \subset X$ при каждом $\lambda \in[0,1]$;

D) многозначные отображения $\partial_{x} \psi(x ; \lambda), \partial_{x} d_{C(\lambda)}(x): X \times[0,1] \rightarrow X^{*}$ полунепрерывны сверху по $\lambda$ равномерно относительно $x$ на каждом шаре $B(r) \subset X$;

Е) для каждого $\lambda \in[0,1]$ и в каждой точке $x$, для которых $\psi(x ; \lambda)=0$, функционал $\psi(x ; \lambda)$ регулярен;

F) задача $(24)-(26)$ при каждом $\lambda \in[0,1]$ имеет экстремаль $x_{*}(\lambda)$, непрерывно зависящую от $\lambda$;

$\mathrm{G)}$ существует $R>0$ такое, что при каждом $\lambda \in[0,1]$ и $x \in B\left(x_{*}(\lambda), R\right) \cap$ $D(\lambda) \backslash\left\{x_{*}(\lambda)\right\}$

$$
0 \notin \partial f(x ; \lambda)+\mu \partial \psi(x ; \lambda)+\alpha \partial d_{C(\lambda)}(x),
$$


где $f(x ; \lambda)=\sup p\left(F(x ; \lambda)-F\left(x_{*}(\lambda) ; \lambda\right)\right)\left(p \in K^{*}(\lambda),|p|_{Y^{*}}=1\right), \mu \geqslant 0, \alpha>0$, $\mu \psi(x ; \lambda)=0$

Н) при каждом $\lambda \in[0,1]$ и $\mu, \alpha>0$ функционал

$$
f(x ; \lambda)+\mu \psi^{+}(x ; \lambda)+\alpha d_{C(\lambda)}(x)
$$

удовлетворяет условию (ПС) на каждом ограниченном множестве в $X, \psi^{+}(x, \lambda)=$ $\max (0, \psi(x, \lambda))$.

ТЕорема 2. Пусть существует невырожденная деформачия задачи (27)-(29) в задачу (30)-(32). Пусть при каждом $\lambda \in[0,1]$ u $\alpha \geqslant 0$ для функиионалов $\psi(x ; \lambda)$ и $d_{C(\lambda)}(x)$ выполнено условие регулярности

$$
0 \notin \partial \psi\left(x_{*}(\lambda), \lambda\right)+\alpha \partial d_{C(\lambda)}\left(x_{*}(\lambda)\right) .
$$

Пусть точка $x_{*}(0)$ является точкой локального $K$-оптимума задачи (27)-(29).

Тогда точка $x_{*}(1)$ является точкой локального $K$-оптимума задачи (30)-(32).

Если функциональ $p(F(x ; 1))+\mu \psi^{+}(x ; 1)+\alpha d_{C(1)}(1) \quad(p \in \mathfrak{P}(1), \mu, \alpha \geqslant 0)$ на каждом шаре удовлетворяют условию $(\Pi C)$ и на $D(1) \cap B\left(x_{*}(1), R\right) \backslash\left\{x_{*}(1)\right\}$ не имеют критических точек, то $x_{*}(1)$ является локально оптимальной точкой задачи (30)-(32).

ДокАЗАТЕЛЬСТВо. Не уменьшая общности, можно считать, что $x_{0}(\lambda) \equiv 0$ и $F(0, \lambda) \equiv 0$ при $\lambda \in[0,1]$. Положим

$$
\begin{aligned}
\varphi(x ; \lambda, \alpha) & =\psi^{+}(x ; \lambda)+\alpha d_{C(\lambda)}(x), \\
\Phi(x ; \lambda, \alpha, k) & =f(x ; \lambda)+k \varphi(x ; \lambda, \alpha) .
\end{aligned}
$$

Тогда $D(\lambda)=\{x \in X: \varphi(x ; \lambda, \alpha)=0\}, \alpha>0$.

Для доказательства первого утверждения теоремы достаточно показать, что при достаточно больших $\alpha$ и $k$ семейство функционалов $\Phi(x ; \lambda, \alpha, k)$ удовлетворяет условиям леммы 2 и при $\lambda=0$ функционал $\Phi(x ; 0, \alpha, k)$ имеет в нуле локальный минимум. Зафиксируем $\lambda \in[0,1]$. Пусть $x_{0} \notin C(\lambda)$ и $y \in \partial d_{C(\lambda)}\left(x_{0}\right)$. Покажем, что $|y|_{X^{*}}=1$. В [12] показано, что коэффициент Липшица у функции расстояния равен 1, и если $C$ выпукло, то и $d_{C}(x)$ выпукла.

Пусть $v \in X$ такой, что $d_{C(\lambda)}\left(x_{0}+v\right)=0,|v|<(1+\varepsilon) d_{C(\lambda)}\left(x_{0}\right)$, где $\varepsilon>0$. Тогда при $0<t<1$

$$
d_{C(\lambda)}\left((1-t) x_{0}+t\left(x_{0}+v\right)\right) \leqslant(1-t) d_{C(\lambda)}\left(x_{0}\right) .
$$

В [12] показано также, что вьпуклая функция регулярна, поэтому из (34) вытекает

$$
d_{C(\lambda)}^{0}\left(x_{0} ; v\right) \leqslant-d_{C(\lambda)}\left(x_{0}\right)<-(1+\varepsilon)^{-1}|v| \text {. }
$$

Из (35) следует, что $|y|_{X^{*}}>(1+\varepsilon)^{-1}$ для произвольного $\varepsilon>0$, т.е. $|y|_{X^{*}}=1$. 
В силу свойства D) справедливо неравенство

$$
\sup _{\substack{y \in \partial \psi(x ; \lambda) \\ x \in B(R) \\ \lambda \in[0,1]}}|y|_{X^{*}}=M_{1}<\infty .
$$

В [12] показано, что для функционала

$$
g(x)=\max _{i}\left(g_{i}(x)\right), \quad i=1, \ldots, n ; \quad I(x)=\left\{i: g_{i}(x)=g(x)\right\},
$$

имеет место включение

$$
\partial g(x) \subset \operatorname{co}\left\{\partial g_{i}(x): i \in I(x)\right\}
$$

В (37) имеет место равенство, если $g_{i}(x)(i \in I(x))$ регулярны в точке $x$. Отсюда и из условий D) и Е) вытекает, что многозначное отображение $\partial \psi^{+}(x ; \lambda)$ : $X \times[0,1] \rightarrow X^{*}$ полунепрерывно сверху по $\lambda$ равномерно относительно $x$ на каждом шаре $B(r) \subset X$. Поэтому из (34)-(37) следует, что при $\alpha-M_{1}=b_{1}>0$

$$
\inf _{\substack{y \in \partial \varphi(x ; \lambda ; \alpha) \\ x \in B(R) \backslash C(\lambda) \\ \lambda \in[0,1]}}|y|_{X^{*}} \geqslant b_{1}
$$

Из условий D) и (33) следует, что сушествует шар $B(\rho)$, для которого при $\alpha=M_{1}+b_{1}$

$$
\inf _{\substack{y \in \partial \psi(x ; \lambda)+\alpha \partial d_{C(\lambda)}(x) \\ x \in B(\rho) \\ \lambda \in[0,1]}}|y|_{X^{*}}=b_{2}>0 .
$$

Так как $B(\rho) \backslash D(\lambda)=(B(\rho) \backslash C(\lambda)) \cup(B(\rho) \backslash Q(\lambda))$, то

$$
\inf _{\substack{y \in \partial \varphi(x ; \lambda ; \alpha) \\ x \in B(\rho) \backslash D(\lambda) \\ \lambda \in[0,1]}}|y|_{X^{*}} \geqslant \min \left(b_{1}, b_{2}\right) \geqslant m_{1}>0 .
$$

Пусть

$$
\sup _{\substack{y \in \partial f(x ; \lambda) \\ x \in B(R) \\ \lambda \in[0,1]}}|y|_{X^{*}}=M_{2} .
$$

Покажем, что при $\alpha=M_{1}+b_{1}$ и $k>3 M_{2} / m_{1}$ выполнены условия леммы 2 для функционалов $\Phi(x ; \lambda, \alpha, k)$. Пусть точка $x=0$ реализует локальный минимум функционала $f(x ; 0)$ на множестве $D(0) \cap B(r), 2 r<\rho<R$. Не ограничивая обшности, можно считать, что точка $x=0$ принадлежит гранище множества $D(0)$. Пусть $\mathfrak{N}$ - множество сплайнов, построенных для функционала $\varphi(x)=\varphi(x ; 0, \alpha)$ и множества $B(2 r) \backslash D(0)$ по той же конструкции, что и сплайны из множества $\mathfrak{M}$, построенного при доказательстве леммы 2 для функционалов $f(x ; \lambda)$ и шара $B\left(R_{1}\right)$. 
Напомним, что на $n$-м звене непрерывного сплайна $x(t) \in \mathfrak{N}$ выполняются соотношения

$$
\begin{gathered}
\dot{x}(t)=v_{n-1}, \quad t_{n-1}<t<t_{n}, \quad\left|v_{n-1}\right|=1, \\
\varphi^{0}\left(x(t) ; v_{n-1}\right) \leqslant-\mu(\varphi ; 3|x(t)| / 4) / 2,
\end{gathered}
$$

где

$$
\mu(\varphi ; s)=\inf _{\substack{y \in \partial \varphi(x) \\ s \leqslant x \leqslant R \\ X \notin D(0)}}|y|_{X^{*}} .
$$

Для того же звена сплайна из (38), (39) вытекает неравенство

$$
\Phi^{0}\left(x ; 0, \alpha, k ; v_{n-1}\right) \leqslant f^{0}\left(x ; 0 ; v_{n-1}\right)+k \varphi^{0}\left(x ; v_{n-1}\right)<0 .
$$

Тогда для функций $\gamma(t)=\Phi(x(t) ; 0, \alpha, k), \eta(t)=\varphi(x(t))$ почти везде справедливы неравенства

$$
\begin{gathered}
\dot{\gamma}(t)<0, \\
\dot{\eta}(t) \leqslant-\mu(\varphi ; 3|x(t)| / 4) / 2 .
\end{gathered}
$$

Если сплайн $x(t)$ начинается в точке $x_{0} \in B(r) \backslash D(0)$, то для него возможны два варианта. Либо сушествует $T$ (конечное или бесконечное) такое, что $x(T) \in$ $B(2 r) \cap D(0)$, либо найдется $T_{1}$ такое, что $\left|x\left(T_{1}\right)\right|=2 r$. В первом случае из (40) следует

$$
\Phi\left(x_{0} ; 0, \alpha, k\right)>f(0 ; 0) .
$$

Во втором случае из (41) вытекает

$$
k \varphi\left(x_{0}\right)>k_{1} r, \quad k_{1}>M_{2} .
$$

Из (43) следует, что

$$
k \varphi\left(x_{0}\right)>k_{1} d_{D(0)}\left(x_{0}\right) .
$$

В [12] показано, что функционал $f(x ; 0)+k_{1} d_{D(0)}(x)$ имеет минимум на шаре $B(r)$ в точке $x=0$. Тогда из (42) и (44) следует $\Phi\left(x_{0} ; 0, \alpha, k\right)>f(0 ; 0)$, т.е. функционал $\Phi(x ; 0, \alpha, k)$ имеет минимум на шаре $B(r)$ в точке $x=0$ при $\alpha=M_{1}+b_{1}$ и $k>3 M_{2} / m_{1}$.

Пусть $\lambda \in[0,1]$ и $0<\rho<R / 2$. В силу (38) получим для $x \in B(\rho) \backslash D(\lambda)$

$$
\inf _{y \in \partial \Phi(x ; \lambda, \alpha, k)}|y|_{X^{*}} \geqslant \inf _{y \in \partial f(x ; \lambda)+k \partial \varphi(x ; \lambda, \alpha)}|y|_{X^{*}} \geqslant k m_{1}-M_{2}>0 .
$$

Если $x \in B(\rho) \cap D(\lambda) \backslash\{0\}$, то $0 \notin \partial \Phi(x ; \lambda, \alpha, k)$ по условию теоремы. Таким образом, условия леммы 2 выполнены и первое утверждение теоремы 2 доказано.

Для доказательства второго утверждения теоремы 2 применим схему доказательства второго утверждения теоремы 1 к функционалу $p(F(x ; 1))+k \varphi(x ; 1, \alpha)$, $p \in \mathfrak{P}(1), \alpha=M_{1}+b_{1}, k>3 M_{2} / m_{1}$. Получим, что сушествует шар $B\left(\rho_{1}\right)$, для ненулевых точек которого $p(F(x ; 1))+k \varphi(x ; 1, \alpha)>0$ для $\forall p \in \mathfrak{P}(1)$, т.е. на множестве $B\left(\rho_{1}\right) \cap D(1) \backslash\{0\}$ имеет место неравенство $p(F(x ; 1))>0$, т.е. $F(x ; 1)>0$. Теорема доказана. 


\section{§4. Достаточные условия для} существования глобального $K$-оптимума

Говорят [13], что локально липшицев функционал $\varphi(x)$ на банаховом пространстве $X$ удовлетворяет слабому условию (ПС), если для любой последовательности $x_{n}, n \in \mathbb{N}$, в $X$, обладающей свойствами

$$
\begin{gathered}
\left|\varphi\left(x_{n}\right)\right| \leqslant \operatorname{const,}_{\inf _{y \in \partial \varphi\left(x_{n}\right)}|y|_{X^{*}} \rightarrow 0,} \notin \partial \varphi\left(x_{n}\right) \quad \forall n,
\end{gathered}
$$

сушествует точка $x_{*} \in X$ такая, что

$$
\liminf \varphi\left(x_{n}\right) \leqslant \varphi\left(x_{*}\right) \leqslant \lim \sup \varphi\left(x_{n}\right), \quad 0 \in \partial \varphi\left(x_{*}\right) .
$$

Для дальнейшего изложения нам потребуется обобщение результата Амброзетти и Рабиновича [13].

Теорема 3. Пусть $X$ - банахово пространство и $\varphi: X \rightarrow \mathbb{R}$ - локально липшичев функиионал, удовлетворяющий на $X$ слабому условию (ПС) $и$

$$
\begin{gathered}
\exists \alpha>0: m(\alpha)=\inf \{\varphi(x):|x|=\alpha\}>\varphi(0), \\
\exists z \in X:|z|>\alpha \quad \text { u } \quad \varphi(z)<m(\alpha) .
\end{gathered}
$$

Тогда существует точка $x_{*} \in X$ такая, что

$$
\varphi\left(x_{*}\right) \geqslant m(\alpha), \quad 0 \in \partial \varphi\left(x_{*}\right) .
$$

ДоКАЗАТЕЛЬСТВо. Обозначим через $\mathfrak{G}$ совокупность всех непрерывных путей $c(t), 0 \leqslant t \leqslant 1$, из нуля в $z$, снабдив это множество расстоянием

$$
d\left(c_{1}, c_{2}\right)=\max \left\{\left|c_{1}(t)-c_{2}(t)\right|: 0 \leqslant t \leqslant 1\right\} .
$$

Мы получили полное метрическое пространство. Рассмотрим функцию $I: \mathfrak{G} \rightarrow \mathbb{R}$ :

$$
I(c)=\max \{\varphi(c(t)): 0 \leqslant t \leqslant 1\} .
$$

Функция $I$ полунепрерывна снизу и ограничена снизу величиной $m(\alpha)$. Из $\varepsilon$ вариационного принципа Экланда [13] следует, что для любого $\varepsilon>0$ найдется траектория $c_{\varepsilon}$ такая, что

$$
\begin{gathered}
I\left(c_{\varepsilon}\right)<\inf \{I(c): c \in \mathfrak{G}\}+\varepsilon, \\
\forall c \in \mathfrak{G} \quad I(c) \geqslant I\left(c_{\varepsilon}\right)-\varepsilon d\left(c, c_{\varepsilon}\right) .
\end{gathered}
$$


Пусть $C^{0}([0,1])$ - банахово пространство непрерывных отображений из $[0,1]$ в $X$ с нормой $|c|_{C}=\max \{|c(t)|: 0 \leqslant t \leqslant 1\}$. Пусть $\gamma \in C^{0}([0,1]),|\gamma|_{C}=1$ и $\gamma(0)=\gamma(1)=0$. Тогда для любого $h \in \mathbb{R}$ справедливо неравенство

$$
I\left(c_{\varepsilon}+h \gamma\right)-I\left(c_{\varepsilon}\right) \geqslant-\varepsilon h .
$$

Функционал $I(c)$ формулой (47) определен на всем пространстве $C^{0}([0,1])$, он локально липшицев и из (48) следует неравенство

$$
I^{0}\left(c_{\varepsilon} ; \gamma\right) \geqslant-\varepsilon
$$

Неравенство (49) равносильно неравенству

$$
\max \left\{\langle\eta, \gamma\rangle: \eta \in \partial I\left(c_{\varepsilon}\right)\right\} \geqslant-\varepsilon .
$$

Пусть $I_{t}(c)=\varphi(c(t))$. Тогда $I(c)=\max \left\{I_{t}(c): 0 \leqslant t \leqslant 1\right\}$ и

$$
I_{t}^{0}(c ; \gamma)=\varphi^{0}(c(t) ; \gamma(t))
$$

Из теоремы о градиенте поточечного максимума [12] следует включение

$$
\partial I\left(c_{\varepsilon}\right) \subset\left\{\int_{0}^{1} \partial I_{t}\left(c_{\varepsilon}\right) \mu(d t): \mu \in P\left(M\left(c_{\varepsilon}\right)\right)\right\},
$$

где $M(c)=\left\{t \in[0,1]: I_{t}(c)=I(c)\right\}, P(S)$ - множество вероятностных мер Радона, сосредоточенных на $S$. Из (50)-(52) следует неравенство

$$
\sup \left\{\int_{0}^{1} \varphi^{0}\left(c_{\varepsilon}(t) ; \gamma(t)\right) \mu(d t): \mu \in P\left(M\left(c_{\varepsilon}\right)\right)\right\} \geqslant-\varepsilon .
$$

Покажем, что найдется точка $t_{0} \in M\left(c_{\varepsilon}\right)$, для которой выполнено неравенство

$$
\inf _{y \in \partial \varphi\left(c_{\varepsilon}\left(t_{0}\right)\right)}|y|_{X^{*}} \leqslant 2 \varepsilon
$$

В предположении противного в силу теоремы отделимости для каждой точки $t \in M\left(c_{\varepsilon}\right)$ найдется $\gamma_{t} \in X,\left|\gamma_{t}\right|=1$, для которого выполнено неравенство

$$
\varphi^{0}\left(c_{\varepsilon}(t), \gamma_{t}\right) \leqslant-3 \varepsilon / 2 .
$$

В силу полунепрерывности сверху обобщенной производной и компактности $M\left(c_{\varepsilon}\right)$ найдется конечная система $\Delta_{i}$ открытых отрезков, покрывающих $M\left(c_{\varepsilon}\right)$, и элементов $\gamma_{i} \in X,\left|\gamma_{i}\right|=1$, таких, что для точек $t$ каждого отрезка $\Delta_{i}$ выполнено неравенство

$$
\varphi^{0}\left(c_{\varepsilon}(t), \gamma_{i}\right) \leqslant-5 \varepsilon / 4
$$

Построим непрерывную кусочно линейную функцию $\gamma(t), \gamma(0)=\gamma(1)=0$, равную $\gamma_{i}$ для тех точек $\Delta_{i}$, которые не принадлежат соседним отрезкам. В силу (55) для этой функции не выполняется неравенство (53). Мы пришли к противоречию. Но тогда в силу слабого условия (ПС) найдется точка $x_{*} \in X$, для которой

$$
\varphi\left(x_{*}\right) \geqslant m(\alpha), \quad 0 \in \partial \varphi\left(x_{*}\right)
$$


ТЕОРема 4. Пусть выполнены условия теоремы 2 и следующие соотношения:

$$
\begin{array}{r}
\sup _{y \in \partial f(x, 1), x \in X \backslash D(1)}|y|_{X^{*}}<\infty, \\
\inf _{y \in \partial \psi(x, 1), x \in C(1) \backslash D(1)}|y|_{X^{*}}>0,
\end{array}
$$

для каждого $x \in D(1) \backslash\left\{x_{*}(1)\right\}$

$$
0 \notin \partial f(x ; 1)+\mu \partial \psi(x ; 1)+\alpha \partial d_{C(1)}(x),
$$

где $\mu \geqslant 0, \quad \alpha>0, \quad \mu \psi(x ; 1)=0$. Пусть для достаточно больших $\mu$ и $\alpha$ функиионал

$$
f(x ; 1)+\mu \psi^{+}(x ; 1)+\alpha d_{C(1)}(x)
$$

удовлетворяет слабому условию (ПС).

Тогда точка $x_{*}(1)$ реализует глобальный $K$-оптимум задачи (30)-(32). Ecли функциональ

$$
p(F(x ; 1))+\mu \psi^{+}(x ; 1)+\alpha d_{C(1)}(x), \quad p \in \mathfrak{P}(1), \quad \mu, \alpha>0,
$$

не имеют критических точек на $D(1) \backslash\left\{x_{*}(1)\right\}$, удовлетворяют условию $($ ПС) на каждом шаре и слабому условию $(\Pi C)$ на $X$, то точка $x_{*}(1)$ реализует глобальный оптимум задачи (30)-(32).

ДокАЗАТЕЛЬСТво. Вследствие теоремы 2 точка $x_{*}(1)$ является точкой локального $K$-оптимума задачи (30)-(32). Из условий теоремы вытекает отсутствие критических точек, кроме $x_{*}(1)$, у функционала

$$
f(x ; 1)+\mu \psi^{+}(x ; 1)+\alpha d_{C(1)}(x)
$$

при достаточно больших $\mu$ и $\alpha$. Отсюда и из теоремы 3 следует первое утверждение теоремы. Второе утверждение доказывается аналогично.

Пусть $\psi(x ; \lambda)=\psi(x), C(\lambda)=C, \lambda \in[0,1]$, функционал $f(x ; 1)$ тот же, что и в $\S 3$, а $f(x ; 0)$ - некоторый липшищевый на каждом шаре функционал.

ТЕОРема 5. Пусть $X$ - рефлексивное пространство и функциональ $f(x ; 0)$, $f(x ; 1), \psi(x)$ слабо полунепрерывны снизу. Пусть выполнены следуюшие условия:

а) семейство оптимизачионных задач

$$
\begin{gathered}
g(x ; \lambda)=(1-\lambda) f(x ; 0)+\lambda f(x ; 1) \rightarrow \min , \\
\psi(x) \leqslant 0 \\
x \in C
\end{gathered}
$$

не имеет экстремалей в $X$, отличных от нуля при $\lambda \in[0,1]$; 
б) обобщенные градиенты $\partial \psi(x)$ и $\partial d_{C}(x)$ удовлетворяют условию регулярности при $x \neq 0$ :

$$
0 \notin \mu \partial \psi(x)+\alpha \partial d_{C}(x), \quad \mu, \alpha>0 ;
$$

в) функционал $f(x ; 0)$ является растущим на допустимом множестве $D=C \cap Q$

$$
\lim _{x \in D,|x| \rightarrow \infty} f(x ; 0)=\infty .
$$

Тогда точка 0 реализует глобальный К-оптимум задачи (30)-(32).

ДокАЗАТЕЛЬСтво. Зафиксируем некоторое $A>0$ и рассмотрим задачу

$$
\begin{gathered}
f(x ; 1) \rightarrow \min , \\
\psi(x) \leqslant 0, \\
x \in C \\
f(x ; 0)-A \leqslant 0 .
\end{gathered}
$$

В силу условий теоремы множество, выделяемое неравенствами $\psi(x) \leqslant 0$ и $f(x ; 0) \leqslant A$ и включением $x \in C$, слабо компактно. Поэтому задача (56)-(59) имеет решение $x_{0}$. Покажем, что $x_{0}=0$. Согласно [12] точка $x_{0}$ является экстремалью задачи (56)-(59), т.е. для нее выполнены соотношения

$$
\begin{gathered}
0 \in \alpha \partial f\left(x_{0} ; 1\right)+\beta \partial f\left(x_{0} ; 0\right)+\mu \partial \psi\left(x_{0}\right)+\nu \partial d_{C}\left(x_{0}\right), \\
\mu \psi\left(x_{0}\right)=0, \\
\beta\left(f\left(x_{0} ; 0\right)-A\right)=0,
\end{gathered}
$$

где $\alpha, \beta, \mu \geqslant 0, \nu>0, \alpha+\beta+\mu>0$.

В силу условия б) справедливо неравенство $\alpha+\beta>0$, и соотношения (60), (61) можно переписать в виде

$$
\begin{gathered}
0 \in\left(1-\lambda_{0}\right) \partial f\left(x_{0} ; 0\right)+\lambda_{0} \partial f\left(x_{0} ; 1\right)+\bar{\mu} \partial \psi\left(x_{0}\right)+\bar{\nu} \partial d_{C}\left(x_{0}\right), \\
\bar{\mu} \psi\left(x_{0}\right)=0,
\end{gathered}
$$

где $\lambda_{0}=\alpha /(\alpha+\beta), \bar{\mu}=\mu /(\alpha+\beta), \bar{\nu}=\nu /(\alpha+\beta)$.

В силу условия а) имеем $x_{0}=0$. Поскольку константа $A$ произвольна, то в силу условия в) 0 - точка глобального $K$-оптимума задачи (30)-(32). Теорема доказана.

ЗАмЕЧАниЕ. Все приведенные теоремы без труда распространяются на случай, когда $Y$ конечномерно, а компоненты вектора $F(x)=\left(f_{1}(x), \ldots, f_{n}(x)\right)$ являются локально липшицевыми функционалами. 


\section{Список литературы}

1. Бобилёв Н. А. О деформационном методе исследования функционалов качества систем с бесконечным числом степеней свободы // Автоматика и телемеханика. 1981. №7. C. $11-13$.

2. Бобылёв Н. А. О деформации функционалов, имеющих единственную критическую точку // Матем. заметки. 1983. Т. 34. № 3. С. 387-398.

3. Бобылёв H. А. Деформационный метод исследования задач нелинейного программирования. I // Автоматика и телемеханика. 1989. №7. С. 82-90.

4. Бобылёв H. А. Деформационный метод исследования задач нелинейного программирования. II // Автоматика и телемеханика. 1989. № 8. С. 24-33.

5. Бобълёв Н. А., Кондаков Г. В. Деформационный метод исследования негладких оптимизационных задач // Автоматика и телемеханика. 1991. № 5. С. 46-57.

6. Барбакадзе Т. Н., Бобъилёв Н. А., Кондаков Г. В. Деформационный принцип минимума для функционалов на банаховых пространствах // Динамика неоднородных систем. Сб. трудов ВНИИСИ. 1991. Вып. 15. С. 6-15.

7. Скалыга В. И. О деформационном методе исследования на условный минимум функционалов качества систем с бесконечным числом степеней свободы // Автоматика и телемеханика. 1991. №6. С. 47-55.

8. СКалыга В. И. Деформационный метод исследования экстремальных задач с ограничениями // Динамика неоднородных систем. Сб. трудов ВНИИСИ. 1991. Вып. 14. С. 59-63.

9. Скалыга В. И. Деформационный метод исследования бесконечномерных оптимизационных задач // Матем. заметки. 1993. Т. 53. № 2. С. 175-176.

10. Скалыга В. И. Деформационный метод исследования негладких бесконечномерных оптимизационных задач // Автоматика и телемеханика. 1993. №11. С. 66-69.

11. Сћальга В.И. О деформациях негладких оптимизационных задач, имеющих изолированную экстремаль // Изв. РАН. Сер. матем. 1994. Т. 58. № 4. С. 186-193.

12. Кларж Ф. Оптимизация и негладкий анализ. М.: Наука, 1988.

13. Обен Ж. П., Экланд И. Прикладной нелинейный анализ. М.: Мир, 1988.

14. Данфорд Н., Швари, Дж. Т. Линейные операторы, общая теория. М.: ИЛ, 1962.

Поступило в редакцию 25.X.1995 